Pacific Journal of Mathematic 


\title{
SINGLY GENERATED ANTISYMMETRIC OPERATOR ALGEBRAS
}

\author{
John B. ConWAy AND WaCŁaW Szymanski
}

\begin{abstract}
We discuss the antisymmetry of certain algebras associated with a bounded linear operator on a Hilbert space $\mathscr{H}$. An algebra of operators on $\mathscr{C}$ is said to be antisymmetric if the only self-adjoint operators it contains are multiples of the identity. If $T$ is a bounded operator on $\mathscr{H}$, let $\mathscr{A}_{u}(T)$ be the norm closure of $\{p(T): p$ is a polynomial $\}$ and let $\mathscr{R}_{u}(T)$ be the norm closure of $\{f(T): f$ is a rational function with poles off the spectrum of $T$. Suppose $T=$ $T_{1} \oplus T_{2} \oplus \cdots$ and $\left\|T_{j}\right\| \rightarrow 0$ as $j \rightarrow \infty$. For a subset $J$ of $N$, the natural numbers, let $T_{J}=\oplus\left\{T_{j}: j \in J\right\}$. It is proved here that if $\mathscr{A}_{u}\left(T_{j}\right)$ is antisymmetric for each $j$, then $\mathscr{A}_{u}(T)$ is antisymmetric if and only if for each finite set $J$ the polynomially convex hulls of $\sigma\left(T_{J}\right)$ and $\sigma\left(T_{N / J}\right)$ have nonempty intersection.
\end{abstract}

Similar results are obtained for $\mathscr{R}_{u}(T)$. Under the assumption that each $\mathscr{A}_{u}\left(T_{j}\right)\left(\mathscr{R}_{u}\left(T_{j}\right)\right)$ is antisymmetric, the maximal antisymmetric projections for $\mathscr{A}_{u}(T)\left(\mathscr{R}_{u}(T)\right)$ are characterized. These results are then applied to completely characterize the compact operators $T$ for which $\mathscr{A}_{u}(T)\left(=\mathscr{R}_{u}(T)\right)$ is antisymmetric.

The concept of antisymmetric algebras has been fruitfully used in the theory of function algebras [4] and operator theory [2], and a systematic study of this property was begun in [5] and [6].

In this paper the cases where $T=T_{1} \oplus \cdots \oplus T_{n}$ or $T=T_{1} \oplus$ $T_{2} \oplus \cdots$ and $\left\|T_{j}\right\| \rightarrow 0$ as $j \rightarrow \infty$, and $\mathscr{A}_{u}\left(T_{j}\right)$ (or $\mathscr{R}_{u}\left(T_{j}\right)$ ) is antisymmetric for each $j \geqq 1$ will be considered. The study of the case where $T$ is an arbitrary infinite direct sum of antisymmetric operators poses some difficulties. The assumption that $\left\|T_{j}\right\| \rightarrow 0$ obviates these difficulties and permits a study of compact operators.

By Theorem 1.4.5 of [1], if $T$ is a compact operator, $T=T_{1} \oplus$ $T_{2} \oplus \cdots$ where each $T_{j}$ is an irreducible compact operator. Because $T_{j}$ is irreducible, $\mathscr{A}_{u}\left(T_{j}\right)$ is antisymmetric [5]. Since $T$ is compact it must be that the above direct sum is either finite or $\left\|T_{j}\right\| \rightarrow 0$ as $j \rightarrow \infty$. Hence, the study of compact operators will be covered by the more general theorems.

The authors would like to thank the referee, William R. Zame, for his valuable remarks. In the original manuscript, only finite direct sums were treated, and the case of infinite direct sums of compact operators was handled with a separate argument. Professor 
Zame observed that the infinite case could be treated provided the assumption was made that the norms converge to zero. He also made several other suggestions that have resulted in more incisive results and more lucid exposition.

1. Preliminary results. If $\mathscr{A}$ is a subalgebra of $\mathscr{B}(\mathscr{H})$, the algebra of all bounded operators on the separable Hilbert space $\mathscr{H}$, and $\mathscr{A}$ contains the identity, a projection $P$ in $\mathscr{A}^{\prime}$, the commutant of $\mathscr{A}$, is antisymmetric for $\mathscr{A}$ if $\mathscr{A}_{P} \equiv\{A \mid P \mathscr{H}: A \in \mathscr{A}\}$ is an antisymmetric subalgebra of $\mathscr{B}(P \mathscr{H}) . A$ maximal antisymmetric projection for $\mathscr{A}$ is an antisymmetric projection for $\mathscr{A}$ that is maximal, for the usual ordering, in the set of all antisymmetric projections. Let $\mathscr{C}(\mathscr{A})$ denote the set of all maximal antisymmetric projections for $\mathscr{A}$. $\mathscr{C}(\mathscr{A})$ is contained in the center of the von Neumann algebra generated by $\mathscr{A}$ [6]. Also, if $Q$ is any antisymmetric projection for $\mathscr{A}$, there is a $P$ in $\mathscr{M}(\mathscr{A})$ such that $Q \leqq P[6]$. Also, if $P \in \mathscr{C}(\mathscr{A})$ and $Q$ is an antisymmetric projection for $\mathscr{A}$ such that $P Q \neq 0$, then $Q \leqq P$ ([5], Proposition 2). Finally, each antisymmetric projection that belongs to $\mathscr{A}$ must be maximal.

The proof of the following result is left to the reader

Proposition 1.1. If $T=\bigoplus\left\{T_{j}: j \geqq 1\right\}$ and $\left\|T_{j}\right\| \rightarrow 0$ as $j \rightarrow \infty$, then

$$
\sigma(T)=\bigcup_{j=1}^{\infty} \sigma\left(T_{j}\right) \cup\{0\} .
$$

Throughout this paper, the following notation will be fixed. $\boldsymbol{R}$ and $C$ stand for the real and complex numbers, and $\square$ denotes the empty set. If $\left\{\mathscr{H}_{j}\right\}$ is a finite or countable sequence of Hilbert spaces, $\mathscr{H}=\bigoplus_{j} \mathscr{H}_{j}$; if $T_{j} \in \mathscr{B}\left(\mathscr{H}_{j}\right), T=\bigoplus_{j} T_{j}$. Let $P_{j}$ denote the projection of $\mathscr{H}$ onto $\mathscr{H}_{j}$. If $J \subseteq N, \mathscr{H}_{J}=\bigoplus\left\{\mathscr{H}_{j}: j \in J\right\}, P_{J}=\bigoplus$ $\left\{P_{j}: j \in J\right\}, T_{J}=\bigoplus\left\{T_{j}: j \in J\right\}$. If $K$ is a compact subset of $C, K^{\wedge}=$ polynomially convex hull of $K$.

2. The antisymmetry of $\mathscr{A}_{u}(T)$. In this section we will study the antisymmetry of $\mathscr{A}_{u}(T)$ under the assumptions that $T$ is a finite direct sum $T=T_{1} \oplus \cdots \oplus T_{n}$ or $T$ is an infinite direct sum $T=$ $T_{1} \oplus T_{2} \oplus \cdots$ and $\left\|T_{j}\right\| \rightarrow 0$; and $\mathscr{A}_{u}\left(T_{j}\right)$ is antisymmetric for each $j \geqq 1$. However, the results and the proofs will only be given in the infinite case. The statements of the results for the finite case and their proofs are left to the reader.

Lemma 2.1. If $J \subseteq N, P_{J} \in \mathscr{A}_{u}(T)$ iff $\sigma\left(T_{J}\right)^{\wedge} \cap \sigma\left(T_{N_{\backslash} J}\right)^{\wedge}=\square$. 
Proof. It is easy to see that $P_{J} \in \mathscr{A}_{u}(T)$ iff $\mathscr{A}_{u}(T)=\mathscr{A}_{u}\left(T_{J}\right) \oplus$ $\mathscr{A}_{u}\left(T_{N_{J}}\right)$. The proof is now completed by applying Theorem 1.4 of [3].

Theorem 2.2. Suppose that $T=T_{1} \oplus T_{2} \oplus \cdots,\left\|T_{j}\right\| \rightarrow 0$ as $j \rightarrow$ $\infty$, and $\mathscr{A}_{u}\left(T_{j}\right)$ is antisymmetric for each $j$. The following conditions are logically equivalent:

(a) $\mathscr{A}_{u}(T)$ is antisymmetric.

(b) For every finite nonempty subset $J$ of $N, P_{J} \notin \mathscr{A}_{u}(T)$.

(c) For every finite nonempty subset $J$ of $N$, $\sigma\left(T_{J}\right)^{\wedge} \cap \sigma\left(T_{N \backslash J}\right)^{\wedge} \neq \square$.

Proof. Clearly (a) implies (b). By Lemma 2.1, (b) and (c) are equivalent.

Now assume that (b) holds, and we will show that $\mathscr{A}_{u}(T)$ must be antisymmetric. Suppose $A \in \mathscr{A}_{u}(T)$ and $A=A^{*}$. Clearly $A=$ $A_{1} \oplus A_{2} \oplus \cdots$ and each $A_{j} \in \mathscr{A}_{u}\left(T_{j}\right)$. Let $\left\{p_{n}\right\}$ be a sequence of polynomials such that

$$
0=\lim _{n \rightarrow \infty}\left\|p_{n}(T)-A\right\| .
$$

Because $\|p(T)\| \geqq \sup \{|p(z)|: z \in \sigma(T)\}$ for any polynomial $p,\left\{p_{n}\right\}$ is a uniformly Cauchy sequence on $\sigma(T)$. In particular, $p_{n}(0) \rightarrow \alpha$, for some scalar $\alpha$.

If $I_{j}$ denotes the identity on the space $\mathscr{H}_{j}$,

$$
\begin{aligned}
\left\|A_{j}-\alpha I_{j}\right\| & \leqq\left\|A_{j}-p_{n}\left(T_{j}\right)\right\|+\left\|p_{n}\left(T_{j}\right)-p_{n}(0) I_{j}\right\|+p_{n}(0) I_{j}-\alpha I_{j} \| \\
& \leqq\left\|A-p_{n}(T)\right\|+\left\|p_{n}\left(T_{j}\right)-p_{n}(0) I_{j}\right\|\left\|p_{n}(0) I_{j}-\alpha I_{j}\right\| .
\end{aligned}
$$

Because $\left\|T_{j}\right\| \rightarrow 0$ as $j \rightarrow \infty,\left\|p\left(T_{j}\right)-p(0) I_{j}\right\| \rightarrow 0$ as $j \rightarrow \infty$ for any polynomial $p$. Thus, the above inequality implies that $\left\|A_{j}-\alpha I_{j}\right\| \rightarrow$ 0 as $j \rightarrow \infty$.

Now $A_{j} \in \mathscr{A}_{u}\left(T_{j}\right)$ and $A_{j}=A_{j}^{*}$. Since $\mathscr{A}_{u}\left(T_{j}\right)$ is antisymmetric, $A_{j}=\alpha_{j} I_{j}$ for some $\alpha_{j}$ in $\boldsymbol{R}$. From the preceding paragraph it follows that $\alpha_{j} \rightarrow \alpha$ as $j \rightarrow \infty$. Suppose $\alpha_{k} \neq \alpha$ for some $k$. Put $J=$ $\left\{j: \alpha_{j}=\alpha_{k}\right\}$. Because $\alpha_{j} \rightarrow \alpha, J$ must be finite. Also $\alpha_{k}$ is an isolated point of the spectrum of $A=\alpha_{1} I_{1} \oplus \alpha_{2} I_{2} \oplus \cdots$. Hence $P_{J} \in \mathscr{A}_{u}(A) \cong$ $\mathscr{A}_{u}(T)$, contradicting (b). Thus $A=\alpha I$.

Notice that if $J$ is an infinite subset of $N$ such that $N \backslash J$ is also infinite, then Proposition 1.1 implies $0 \in \sigma\left(T_{J}\right) \cap \sigma\left(T_{N \backslash J}\right)$.

The remainder of this section is devoted to the description of $\mathscr{C}\left(\mathscr{A}_{u}(T)\right)$, the set of maximal antisymmetric projections for $\mathscr{A}_{u}(T)$. Let $\mathscr{P}$ denote the collection of nonempty subsets $J$ of $\boldsymbol{N}$ such that $\mathscr{A}_{u}\left(T_{J}\right)$ is antisymmetric. It follows from Theorem 2.2 that 


$$
J \in \mathscr{P} \text { iff } \sigma\left(T_{L}\right)^{\wedge} \cap \sigma\left(T_{J \backslash L}\right)^{\wedge} \neq \square
$$

for every finite nonempty subset $L$ of $J$.

It follows from (2.3) that if $\mathscr{P}$ is ordered by inclusion, each member of $\mathscr{P}$ is contained in a maximal element of $\mathscr{P}$. Let $\mathscr{K}=$ the collection of maximal elements of $\mathscr{P}$. Because each $\mathscr{A}_{u}\left(T_{j}\right)$ is assumed to be antisymmetric, each integer belongs to at least one member of $\mathscr{K}$. Moreover, the sets belonging to $\mathscr{K}$ are pairwise disjoint. Hence, $\mathscr{K}$ is a partition of $N$ and $I=\Sigma\left\{P_{J}: J \in \mathscr{K}\right\}$.

Proposition 2.4. If $\mathscr{K}$ is the partition of $\boldsymbol{N}$ described above, then:

(a) For $J$ and $K$ in $\mathscr{K}, J \neq K, \sigma\left(T_{J}\right)^{\wedge} \cap \sigma\left(T_{K}\right)^{\wedge}=\square$.

(b) If $J \in \mathscr{K}$ and $0 \notin \sigma\left(T_{J}\right)^{\wedge}$, then $P_{J} \in \mathscr{A}_{u}(T)$.

(c) $\mathscr{K}$ contains at most one infinite set $J$, and this infinite set is the only one for which $0 \in \sigma\left(T_{J}\right)^{\wedge}$.

Proof. (a) Suppose $J$ and $K$ belong to $\mathscr{K}$ and $\sigma\left(T_{J}\right)^{\wedge} \cap \sigma\left(T_{K}\right)^{\wedge} \neq$ $\square$. Let $L$ be a finite subset of $J \cup K$ and assume that $L \cap K \neq \square$. Thus $\sigma\left(T_{(J \cup K) \backslash L}\right)^{\wedge} \cap \sigma\left(T_{L}\right)^{\wedge} \supseteqq \sigma\left(T_{K \backslash L}\right)^{\wedge} \cap \sigma\left(T_{L}\right)^{\wedge} \neq \square . \quad$ By (2.3), $J \cup K \epsilon$ $\mathscr{P}$. By the maximality of $J$ and $K, J=K$.

(b) If $0 \notin \sigma\left(T_{J}\right)^{\wedge}$, there is an $\varepsilon>0$ such that for $\Delta=\{z \in C:|z| \leqq \varepsilon\}$, $\Delta \cap \sigma\left(T_{J}\right)^{\wedge}=\square$. Because $\left\|T_{j}\right\| \rightarrow 0$ as $j \rightarrow \infty$, Proposition 1.1 implies that there are a finite number of sets $J_{0}, \cdots, J_{n}$ in $\mathscr{K}$ such that if $K \in \mathscr{C}$ and $K \neq J_{0}, \cdots, J_{n}$, then $\sigma\left(T_{K}\right) \subseteq \Delta$; hence, $\sigma\left(T_{K}\right)^{\wedge} \subseteq \Delta$ for $K \neq J_{0}, \cdots, J_{n}$. Suppose $J=J_{0}$. By $(\mathrm{a}), \quad \sigma\left(T_{N \backslash J}\right)^{\wedge} \subseteq \sigma\left(T_{J_{1}}\right)^{\wedge} \cup \cdots \cup$ $\sigma\left(T_{J_{n}}\right)^{\wedge} \cup \Delta$, and so $\sigma\left(T_{J}\right)^{\wedge} \cap \sigma\left(T_{N \backslash J}\right)^{\wedge}=\square$. Part (b) follows by Lemma 1.1. Part (c) follows from (a) and the fact that $0 \in \sigma\left(T_{j}\right)$ if $J$ is infinite.

THEOREM 2.5. If $\mathscr{A}_{u}\left(T_{j}\right)$ is antisymmetric for each $j \geqq 1$ and $\mathscr{K}$ is the partition of $N$ described above, then

$$
\mathscr{L}\left(\mathscr{A}_{u}(T)\right)=\left\{P_{J}: J \in \mathscr{K}\right\} .
$$

Proof. By the definition of $\mathscr{K}, P_{J}$ is an antisymmetric projection for each $J$ in $\mathscr{K}$. Also note that $\mathscr{A}_{u}(T)_{P_{J}} \subseteq \mathscr{A}_{u}\left(T_{J}\right)$. Let $L$ be the unique element of $\mathscr{K}$ such that $0 \in \sigma\left(T_{L}\right)$. By $2.4(\mathrm{~b}), P_{J} \in$ $\mathscr{A}_{u}(T)$ for $J$ in $\mathscr{K}$ and $J \neq L$; since $P_{J}$ is an antisymmetric projection, it follows, as noted in the remarks at the beginning of $\S 1$, that $P_{J} \in \mathscr{M}\left(\mathscr{A}_{u}(T)\right)$.

Now let $E \in \mathscr{C}\left(\mathscr{A}_{u}(T)\right)$. Because $\sum\left\{P_{J}: J \in \mathscr{K}\right\}=I$, there is a $J$ in $\mathscr{K}$ such that $E P_{J} \neq 0$. By the remarks at the beginning of $\S 1, P_{J} \leqq E$. If $J \neq L$, then $E=P_{J}$ since $P_{J}$ is also maximal. If 
$E \neq P_{J}$ for all $J$ in $\mathscr{K}$ different from $L$, then $E P_{J}=0$ for $J$ in $\mathscr{K}, J \neq L$. Hence, $I-P_{L}=\Sigma\left\{P_{J}: J \in \mathscr{K}, J \neq L\right\} \leqq I-E$, so $E \leqq$ $P_{L}$. Because $P_{L}$ is antisymmetric and $E$ is maximal, $E=P_{L}$.

We have shown that $\mathscr{C}\left(\mathscr{A}_{u}(T)\right) \subseteq\left\{P_{J}: J \in \mathscr{K}\right\}$. It only remains to show that $P_{L}$ is a maximal antisymmetric projection. But this is easy since the fact that it is antisymmetric implies there is an $E$ in $\mathscr{C}\left(\mathscr{A}_{u}(T)\right)$ such that $P_{L} \leqq E$ ([6]). By the preceding paragraph, $P_{L}=E$.

Observe that if $L$ is as in the preceding proof, then $P_{L}$ is not necessarily in $\mathscr{A}_{u}(T)$.

If $T$ is an irreducible operator (that is, $\{T\}^{\prime}$ contains no nontrivial projections), then $\mathscr{A}_{u}(T)$ is antisymmetric [5]. Now if $T$ is compact, $T=T_{1} \oplus T_{2} \oplus \cdots$ where each $T_{j}$ is irreducible ([1], 1.4.5). A combination of this result and Theorem 2.2 yield a description of those compact operators $T$ such that $\mathscr{A}_{u}(T)$ is antisymmetric.

THEOREM 2.6. If $T$ is a compact operator and $T=T_{1} \oplus T_{2} \oplus \cdots$ where each $T_{j}$ is irreducible, then $\mathscr{A}_{u}(T)$ is antisymmetric iff for each finite subset $J$ of $N, \sigma\left(T_{J}\right) \cap \sigma\left(T_{N \backslash J}\right) \neq \square$.

Note that because the spectrum of a compact operator is polynomially convex, it is not necessary to use the polynomially convex hulls of the spectra in Theorem 2.6. Also, this same fact implies

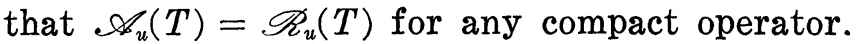

COROLLARY 2.7. If $T$ is a compact operator and $T=T_{1} \oplus$ $T_{2} \oplus \cdots$, where each $T_{j}$ is irreducible and has infinite rank, then $\mathscr{A}_{u}(T)$ is antisymmetric.

The following example illustrates Theorem 2.6.

EXAMPLE 2.8. Consider

$$
T_{n}=\left[\begin{array}{cc}
\frac{1}{n} & 0 \\
\frac{1}{n} & \frac{1}{n+1}
\end{array}\right]
$$

acting on $C^{2}$ for $n=1,2, \cdots$. Also let $V$ be the Volterra operator on $L^{2}[0,1]$ :

$$
V f(x)=\int_{0}^{x} f(t) d t
$$

Then $V$ and $T_{n}$ are irreducible and $\left\|T_{n}\right\| \rightarrow 0$. Therefore, $T=$ 
$\bigoplus_{n=1}^{\infty} T_{n} \oplus V$ is a compact operator. Now $\sigma\left(T_{n}\right)=\{1 / n, 1 /(n+1)\}$ so if $T_{0}=\bigoplus_{n=1}^{\infty} T_{n}$, then $\mathscr{A}_{n}\left(T_{0}\right)$ is antisymmetric by Theorem 2.6. Also, $0 \in \sigma\left(T_{0}\right)$ so that $\mathscr{A}_{u}(T)$ is antisymmetric by Theorem 2.2 .

This section concludes with a word of caution to the reader. If $J \in \mathscr{K}$, the partition constructed for Theorem 2.5, then $\mathscr{A}_{u}\left(T_{J}\right)$ is antisymmetric and $P_{J}$ is an antisymmetric projection for $\mathscr{A}_{u}(T)$. However, if $J$ is an arbitrary subset of $N$ and $P_{J}$ is an antisymmetric projection for $\mathscr{A}_{u}(T)$, it does not follow that $\mathscr{A}_{u}\left(T_{J}\right)$ is antisymmetric. This is illustrated by the following example.

Let $X_{1}=\{z \in C:|z| \leqq 1\} \cap\{z \in C: \operatorname{Re} z \leqq 0\}, \quad X_{2}=\{1 / 2\}, X_{3}=\{z \in$ $C:|z|=1\}$. Let $T_{j}$ be a normal operator with $\sigma\left(T_{j}\right)=X_{j}, j=1,2,3$. Put $T=T_{1} \oplus T_{2} \oplus T_{3}, J=\{1,2\}$. Now $\mathscr{A}_{u}\left(T_{J}\right)=\mathscr{A}_{u}\left(T_{1}\right) \oplus \mathscr{A}_{u}\left(T_{2}\right)$ since $X_{1} \cap X_{2}=X_{1}^{\wedge} \cap X_{2}^{\wedge}=\square$. Hence $\mathscr{A}_{u}\left(T_{J}\right)$ is not antisymmetric. On the other hand, suppose $A \in \mathscr{A}_{u}(T)$ and $A P_{J}=A^{*} P_{J}$. Let $\left\{p_{n}\right\}$ be a sequence of polynomials such that $p_{n}(T) \rightarrow A$ as $n \rightarrow \infty$. Because $T$ is normal, there is a continuous function $f_{0}$ on $\sigma(T)=X_{1} \cup X_{2} \cup$ $X_{3}$, such that $p_{n} \rightarrow f_{0}$ uniformly on $\sigma(T)$ and $A=f_{0}(T)$. But by the Maximum Modulus Theorem, $\left\{p_{n}\right\}$ converges uniformly on the closed unit disk $D$ to a function $f$ that is continuous on $D$ and analytic on its interior. Clearly $f=f_{0}$ on $\sigma(T)$. But $f$ must be real-valued on $X_{1}$, so $f$ is a constant function, say $f \equiv c$. Hence $A=f_{0}(T)=c I$, and $\mathscr{A}_{u}(T)_{P_{J}}$ is antisymmetric.

In the preceding example, $\mathscr{C}\left(\mathscr{A}_{u}(T)\right)=\{I\}$, since $\mathscr{A}_{u}(T)$ is antisymmetric.

3. The antisymmetry of $\mathscr{R}_{u}(T)$. Here we assume that $T$ is a finite direct sum or an infinite direct sum of operators $\left\{T_{j}\right\}$ such that $\left\|T_{j}\right\| \rightarrow 0$ as $j \rightarrow \infty$, and $\mathscr{R}_{u}\left(T_{j}\right)$ is antisymmetric for each $j$. As in $\S 2$, only the results for infinite direct sums will be given. In fact, no proofs of results analogous to results in $\S 2$ are given here; these proofs following by analogous arguments.

Lemma 3.1. If $J \subset N, P_{J} \in \mathscr{R}_{u}(T)$ iff $\sigma\left(T_{J}\right) \cap \sigma\left(T_{N \backslash J}\right)=\square$.

This is proved like Lemma 2.1, but Theorem 2.2 of [3] is invoked. The proof of the next theorem is similar to the proof of Theorem 2.2.

THEOREM 3.2. Suppose that $T=T_{1} \oplus T_{2} \oplus \cdots,\left\|T_{j}\right\| \rightarrow 0$ as $j \rightarrow$ $\infty$, and $\mathscr{R}_{u}\left(T_{j}\right)$ is antisymmetric for each $j$. The following conditions are logically equivalent:

(a) $\mathscr{R}_{u}(T)$ is antisymmetric.

(b) For every finite nonempty subset $J$ of $N, P_{J} \notin \mathscr{R}_{u}(T)$.

(c) For every finite nonempty subset $J$ of $N$, 
$\sigma\left(T_{J}\right) \cap \sigma\left(T_{M \backslash J}\right) \neq \square$

Because $\mathscr{R}_{u}(T) \supset \mathscr{A}_{u}(T)$, if $\mathscr{R}_{u}(T)$ is antisymmetric, $\mathscr{A}_{u}(T)$ is antisymmetric. However, the converse is false as the next example illustrates.

EXAMPLE 3.3. Consider the following three compact subsets of the plane: $K_{1}=\{z:|z| \leqq 1\}, K_{2}=\{z: 9 \leqq|z-10| \leqq 10\} \mid\{z:|\operatorname{Im} z|<1 / 2$ and $|z|<2\}$, and $K_{3}=\{z:|z-5| \leqq 1\}$. Let $U_{i}=\operatorname{int} K_{i}$ and let $\lambda_{i}=$ planar Lebesgue measure restricted to $U_{i}$. Let $A^{2}\left(U_{i}\right)=\{f: f$ is an analytic function on $U_{i}$ with $\left.\int|f|^{2} d \lambda_{i}<\infty\right\}$ and define $T_{i}$ on $A^{2}\left(U_{i}\right)$ by $T_{i} f=z f$. It follows that $\mathscr{A}_{u}\left(T_{i}\right)=\mathscr{R}_{u}\left(T_{i}\right)=\left\{M_{\phi}: \phi\right.$ is a continuous function on $K_{i}$ that is analytic on $\left.U_{i}\right\}$, where $M_{\phi} f=\phi f$. Moreover $\sigma\left(T_{i}\right)=K_{i}=K_{i}$. Let $T=T_{1} \oplus T_{2} \oplus T_{3}$. By Theorem 2.2 $\mathscr{A}_{u}(T)$ is antisymmetric. However $\sigma\left(T_{3}\right) \cap\left[\sigma\left(T_{2}\right) \cup \sigma\left(T_{1}\right)\right]=\square$ so that $\mathscr{R}_{u}(T)$ is not antisymmetric by Theorem 3.2.

To describe $\mathscr{N}\left(\mathscr{R}_{u}(T)\right)$, let $\mathscr{P}=\left\{J \subseteq N: \mathscr{R}_{u}\left(T_{J}\right)\right.$ is antisymmetric $\}$ and let $\mathscr{K}$ be the maximal elements of $\mathscr{P}$. The next result is proved like Theorem 2.5.

THEOREM 3.4. If $\mathscr{R}_{u}\left(T_{j}\right)$ is antisymmetric for each $j \geqq 1$ and $\mathscr{K}$ is the partition of $N$ described above,

$$
\mathscr{C}\left(\mathscr{R}_{u}(T)\right)=\left\{P_{J}: J \in \mathscr{K}\right\} .
$$

4. Final remarks. There are some situations in which $\mathscr{A}_{u}(T \oplus$ $S)$ or $\mathscr{R}_{u}(T \oplus S)$ is antisymmetric even though $\mathscr{A}_{u}(T)$ and $\mathscr{A}_{u}(S)$ or $\mathscr{R}_{u}(T)$ and $\mathscr{R}_{u}(S)$ are not.

Proposition 4.1. Let $T \in \mathscr{L}\left(\mathscr{H}_{T}\right)$ and $S \in \mathscr{L}\left(\mathscr{H}_{S}\right)$.

(a) If $\mathscr{A}_{u}(S)$ is antisymmetric, $\sigma(T)^{\wedge} \subseteq \sigma(S)^{\wedge}$, and $\sigma(T)^{\wedge}$ is a spectral set for $T$, then $\mathscr{A}_{u}(T \oplus S)$ is antisymmetric.

(b) If $\mathscr{R}_{u}(S)$ is antisymmetric, $\sigma(T) \leqq \sigma(S)$, and $\sigma(T)$ is a spectral set for $T$, then $\mathscr{R}_{u}(T \oplus S)$ is antisymmetric.

Proof. (a) If $A=A^{*} \in \mathscr{A}_{u}(T \oplus S)$, then there is a sequence $\left\{p_{n}\right\}$ of polynomials such that $p_{n}(T \oplus S)=p_{n}(T) \oplus p_{n}(S) \rightarrow A$. Hence $A=A_{1} \oplus A_{2}$ where $A_{1}$ and $A_{2}$ are self-adjoint, $p_{n}(T) \rightarrow A_{1}$, and $p_{n}(S) \rightarrow A_{2}$. Since $\mathscr{A}_{n}(S)$ is antisymmetric, $A_{2}=c I$. It follows that $p_{n}(z) \rightarrow c$ uniformly on $\sigma(T)^{\wedge}$. But $\sigma(T)^{\wedge}$ is a spectral set for $T$, so $\left\|p_{n}(T)-c I\right\| \leqq \sup \left\{\left|p_{n}(z)-c\right|: z \in \sigma(T)^{\wedge}\right\}$. Thus $p_{n}(T) \rightarrow c I$ and $A_{1}=c I$.

(b) The proof of (b) is similar.

COROLlaRY 4.2. If $\mathscr{A}_{u}(S)$ [resp., $\left.\mathscr{R}_{u}(S)\right]$ is antisymmetric and 
$T$ is a subnormal operator with $\sigma(T)^{\wedge} \subset \sigma(S)^{\wedge}[$ resp., $\sigma(T) \subset \sigma(S)]$, then $\mathscr{A}_{u}(T \oplus S)$ [resp., $\left.\mathscr{R}_{u}(T \oplus S)\right]$ is antisymmetric.

CoROLlaRy 4.3. If $\mathscr{H}_{u}(S)$ [resp., $\left.\mathscr{R}_{u}(S)\right]$ is antisymmetric, $T$ is a contraction, and $\{z:|z| \leqq 1\} \subset \sigma(S)^{\wedge}[$ resp., $\{z:|z| \leqq 1\} \subset \sigma(S)]$, then $\mathscr{A}_{u}(T \oplus S)$ [resp., $\left.\mathscr{R}_{u}(T \oplus S)\right]$ is antisymmetric.

These ideas have a nice application to compact operators

CoROLlaRy 4.4. If $T$ and $N$ are compact operators and $N$ is normal, then $\mathscr{A}_{u}(T \oplus N)$ is antisymmetric if and only if $\mathscr{A}_{u}(T)$ is antisymmetric and $\sigma(N) \subset \sigma(T)$.

Proof. If $\mathscr{A}_{u}(T)$ is antisymmetric and $\sigma(N) \subset \sigma(T)$, then $\mathscr{A}_{u}(T \oplus N)$ is antisymmetric by Corollary 4.2. Now assume that $\mathscr{A}_{n}(T \oplus N)$ is antisymmetric. Let $T=\bigoplus_{n=1}^{\infty} T_{n}$ where each $T_{n}$ is irreducible and let $N=\bigoplus_{n=1}^{\infty} \lambda_{n}$ where $\left\{\lambda_{1}, \lambda_{2}, \cdots\right\}$ are the eigenvalues of $N$, each repeated according to its multiplicity; each $\lambda_{n}$ is considered as acting on a one dimensional Hilbert space. Then

$$
T \bigoplus N=\bigoplus_{n=1}^{\infty} T_{n} \oplus \bigoplus_{n=1}^{\infty} \lambda_{n}
$$

is the decomposition of the compact operator $T \oplus N$ as a direct sum of irreducible compact operators. Fix an integer $k$ and let $J=$ $\left\{j \in N ; \lambda_{j}=\lambda_{k}\right\}$ : Applying (2.6) to this $J$, it follows that $\lambda_{k} \in \sigma(T \oplus$ $\left.\bigoplus_{j \notin J} \lambda_{j}\right)=\sigma(T) \cup\left\{\lambda_{j}: j \notin J\right\}=\sigma(T) \cup\left\{\lambda_{j}: j \notin J\right\} \cup\{0\}$. Clearly if $\lambda_{k} \neq 0$ then $\lambda_{k} \in \sigma(T)$. So $\sigma(N) \backslash\{0\} \subseteq \sigma(T)$. If $\sigma(N)$ is infinite, then $\sigma(T)$ must be infinite and so $0 \in \sigma(T)$. If $\sigma(N)$ is finite and $\lambda_{k}=0$, then (2.6) gives that $0 \in \sigma(T) \cup\left\{\lambda_{j}: j \neq J\right\}$, so $0 \in \sigma(T)$.

\section{REFERENCES}

1. W. Arveson, An Invitation to $C^{*}$-algebras, Springer-Verlag, GTM vol. 39, New York, 1976.

2. J. B. Conway and R. F. Olin, A functional calculus for subnormal operators, II, Memoirs Amer. Math. Soc., no. 184.

3. J. B. Conway and P. Y. Wu, The splitting of $A\left(T_{1} \oplus T_{2}\right)$ and rlated questions, Indiana Math. J., 26 (1977), 41-56.

4. T. W. Gamelin, Uniform algebras, Prentice-Hall, Inc., Englewood Cliffs, N. J. 1969.

5. W. Szymanski, Antisymmetric operator algebras, I, Annales Polon. Math., (to appear).

6. — Antisymmetric operator algebras, II, Annales Polon. Math., (to appear).

Received September 25, 1978 and in revised form May 11, 1979. 
INDIANA UNIVERSITY

BLOOMINGTON, IN 47401

AND

Polish ACademy of Sciences

KRAKOW BRANCH

AND

Centro de Investigacion DeL I. P. N.

MeXico 14, D. F. 



\section{PACIFIC JOURNAL OF MATHEMATICS}

\section{EDITORS}

DONALD BABBITT (Managing Editor)

University of California

Los Angeles, CA 90024

HUGo RossI

University of Utah

Salt Lake City, UT 84112

C. C. MOORE and ANDREW OGG

University of California

Berkeley, CA 94720
J. DugundJI

Department of Mathematics

University of Southern California

Los Angeles, CA 90007

R. FinN and J. Milgram

Stanford University

Stanford, CA 94305

ASSOCIATE EDITORS
E. F. BECKENBACH
B. H. NeUmanN
F. WOLF
K. YoSHIDA

\section{SUPPORTING INSTITUTIONS}

UNIVERSITY OF BRITISH COLUMBIA

CALIFORNIA INSTITUTE OF TECHNOLOGY

UNIVERSITY OF CALIFORNIA

MONTANA STATE UNIVERSITY

UNIVERSITY OF NEVADA, RENO

NEW MEXICO STATE UNIVERSITY

OREGON STATE UNIVERSITY

UNIVERSITY OF OREGON
UNIVERSITY OF SOUTHERN CALIFORNIA

STANFORD UNIVERSITY

UNIVERSITY OF HAWAII

UNIVERSITY OF TOKYO

UNIVERSITY OF UTAH

WASHINGTON STATE UNIVERSITY

UNIVERSITY OF WASHINGTON

The Supporting Institutions listed above contribute to the cost of publication of this Journal, but they are not owners or publishers and have no responsibility for its content or policies.

Mathematical papers intended for publication in the Pacific Journal of Mathematics should be in typed form or offset-reproduced, (not dittoed), double spaced with large margins. Please do not use built up fractions in the text of the manuscript. However, you may use them in the displayed equations. Underline Greek letters in red, German in green, and script in blue. The first paragraph or two must be capable of being used separately as a synopsis of the entire paper. Please propose a heading for the odd numbered pages of less than 35 characters. Manuscripts, in triplicate, may be sent to any one of the editors. Please classify according to the scheme of Math. Reviews, Index to Vol. 39. Supply name and address of author to whom proofs should be sent. All other communications should be addressed to the managing editor, or Elaine Barth, University of California, Los Angeles, California, 90024.

50 reprints to each author are provided free for each article, only if page charges have been substantially paid. Additional copies may be obtained at cost in multiples of 50 .

The Pacific Journal of Mathematics is issued monthly as of January 1966. Regular subscription rate: $\$ 84.00$ a year (6 Vols., 12 issues). Special rato: $\$ 42.00$ a year to individual members of supporting institutions.

Subscriptions, orders for numbers issued in the last three calendar years, and changes of address shoud be sent to Pacific Journal of Mathematics, P.O. Box 969, Carmel Valley, CA 93924, U.S.A Old back numbers obtainable from Kraus Periodicals Co., Route 100, Millwood, NY 10546.

PUBLISHED BY PACIFIC JOURNAL OF MATHEMATICS, A NON-PROFIT CORPORATION

Printed at Kokusai Bunken Insatsusha (International Academic Printing Co., Ltd.). 8-8, 3-chome, Takadanobaba, Shinjuku-ku, Tokyo 160, Japan.

Copyright (C) 1980 by Pacific Jounal of Mathematics Manufactured and first issued in Japan 


\section{Pacific Journal of Mathematics \\ Vol. 89, No. $2 \quad$ June, 1980}

Frank Hayne Beatrous, Jr. and R. Michael Range, On holomorphic

approximation in weakly pseudoconvex domains................. 249

Lawrence Victor Berman, Quadratic forms and power series fields ...... 257

John Bligh Conway and Wacław Szymański, Singly generated antisymmetric operator algebras ....................... 269

Patrick C. Endicott and J. Wolfgang Smith, A homology spectral sequence for submersions . . . .................................

Sushil Jajodia, Homotopy classification of lens spaces for one-relator groups with torsion ................................ 301

Herbert Meyer Kamowitz, Compact endomorphisms of Banach

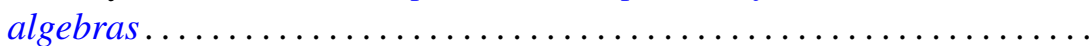

Keith Milo Kendig, Moiré phenomena in algebraic geometry: polynomial

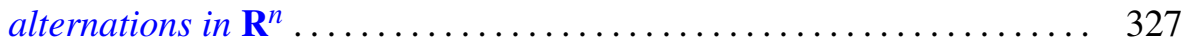

Cecelia Laurie, Invariant subspace lattices and compact operators....... 351

Ronald Leslie Lipsman, Restrictions of principal series to a real form . . . . . 367

Douglas C. McMahon and Louis Jack Nachman, An intrinsic

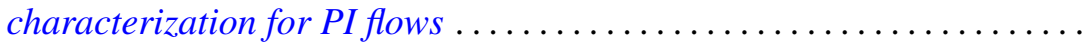

Norman R. Reilly, Modular sublattices of the lattice of varieties of inverse semigroups .................................... 405

Jeffrey Arthur Rosoff, Effective divisor classes and blowings-up of $\mathbf{P}^{2}$ 419

Zalman Rubinstein, Solution of the middle coefficient problem for certain

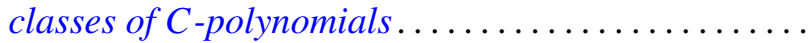

Alladi Sitaram, An analogue of the Wiener-Tauberian theorem for spherical transforms on semisimple Lie groups ................

Hal Leslie Smith, A note on disconjugacy for second order systems ...

J. Wolfgang Smith, Fiber homology and orientability of maps ...

Audrey Anne Terras, Integral formulas and integral tests for series of positive matrices. 